\title{
Outcomes of a virtual-reality simulator-training programme on basic surgical skills in robot- assisted laparoscopic surgery
}

\author{
Véronique Phé ${ }^{1 *}$ \\ Susanna Cattarino ${ }^{1}$ \\ Jérôme Parra ${ }^{1}$ \\ Marc-Olivier Bitker ${ }^{1}$ \\ Vanina Ambrogi ${ }^{2}$ \\ Christophe Vaessen ${ }^{1}$ \\ Morgan Rouprêt ${ }^{1}$ \\ ${ }^{1}$ Pitié-Salpêtrière Academic Hospital, \\ Department of Urology, Assistance \\ Publique-Hôpitaux de Paris, Pierre \\ and Marie Curie Medical School, Paris \\ 6 University, Paris, France \\ ${ }^{2}$ Pitié-Salpêtrière Academic Hospital, \\ Department of Statistics, Assistance \\ Publique-Hôpitaux de Paris, Pierre \\ and Marie Curie Medical School, Paris \\ 6 University, Paris, France \\ *Correspondence to: Véronique Phé, \\ Department of Urology, Pitié- \\ Salpêtrière Academic Hospital, 47-83 \\ boulevard de l'hôpital, 75651 Paris \\ Cedex 13, France. \\ E-mail: veronique.phe@aphp.fr
}

Accepted: 1 February 2016

\begin{abstract}
Background The utility of the virtual-reality robotic simulator in training programmes has not been clearly evaluated. Our aim was to evaluate the impact of a virtual-reality robotic simulator-training programme on basic surgical skills.
\end{abstract}

Methods A simulator-training programme in robotic surgery, using the da Vinci Skills Simulator, was evaluated in a population including junior and seasoned surgeons, and non-physicians. Their performances on robotic dots and suturing-skin pod platforms before and after virtual-simulation training were rated anonymously by surgeons experienced in robotics.

Results 39 participants were enrolled: 14 medical students and residents in surgery, 14 seasoned surgeons, 11 non-physicians. Junior and seasoned surgeons' performances on platforms were not significantly improved after virtual-reality robotic simulation in any of the skill domains, in contrast to non-physicians.

Conclusions The benefits of virtual-reality simulator training on several tasks to basic skills in robotic surgery were not obvious among surgeons in our initial and early experience with the simulator. Copyright (C) 2016 John Wiley \& Sons, Ltd.

Keywords medical subject headings (MeSH): robotic; surgery; learning-curve; simulation

\section{Introduction}

Despite the development of robot-assisted laparoscopic urologic procedures during the last decade, there is no current certified training programme for surgeons (1). During robotic surgery, surgeons perform the procedure without tactile feedback and are physically distant from the operative field, which makes it challenging to learn each step of the procedure from a senior surgeon, in contrast to open surgery or laparoscopic approaches. Residents are expected to overcome learning curves on this new surgical approach without increasing the morbidity of the procedure (2). Although it has been established that it is easier to overcome learning curves in robotic surgery than in laparoscopic 
surgery, several training methods have been proposed to support operative experience during robotic surgery, notably the use of simulation $(3,4)$.

There is a wide range of simulators available for urological procedures (3). Studies of simulation in laparoscopic procedures conclude that a structured programme could improve performance and patient safety in the operating room (5-7). In contrast, the methodologies to shorten learning curves in robotic surgery have not been clearly evaluated.

Although increased robotic experience decreases complication rates in robotic prostatectomies (8), a resident's exposure to console surgery may be limited (9). Virtualreality (VR) robotic-simulation training has been proposed to help residents to improve their skills before 'live' surgery $(10,11)$. VR simulators have the advantage of reusability and provide statistical feedback through an objective performance-evaluation report.

The daVinci Skills Simulator has been tested for face, content, construct, concurrent, and predictive validity (10-13). Most of the exercises of the da Vinci Skills Simulator (dVSS, Intuitive Surgical Inc., Sunnyvale, CA, USA) for the da Vinci Si version are based on software developed by Mimic Technologies (Seattle, WA, USA). However, in contrast to VR laparoscopic simulators, the utility of the VR robotic simulator in training programmes has not been clearly evaluated.

\section{Objectives}

Our purpose was to evaluate the impact of a VR roboticsimulator training programme on basic surgical skills in a population of beginner and seasoned surgeons.

\section{Materials and methods}

This monocentric prospective study was conducted in our department of urology.

\section{Participants}

After informed consent and institutional review approval, a population of potential operators was recruited to conduct surgical exercises before and after taking the VR simulator training programme: medical students, residents in surgery, chief residents in urology, senior surgeons and non-physicians were invited to attend a VR simulator training programme in robotic surgery using the dVSS. Medical students and residents in surgery were placed within the 'junior surgeon group': they had no experience in robotic surgery. Senior surgeons and chief residents were placed in the 'seasoned surgeon group'. The non-physicians were engineering students totally naïve of any surgical or medical procedures.

\section{Description of the training platforms}

The dry-laboratory training set-up comprised a robotic suturing-skin pod and a robotic-dot platform (The Chamberlain Group, Massachusetts, USA) (Figure 1).

On the robotic-dot platform, using a da Vinci robot, users had to pass a threaded needle into different holes in a concentric circle. On the robotic suturing-skin pod, users had to perform a continuous straight-line suture and make knots at the two extremities of the suture.

The dVSS uses software developed by Mimic technologies. The platforms contain a variety of exercises designed to reach proficiency with the da Vinci console controls and in basic surgical skills. The portable case, or 'backpack', which has dimensions of $57.2 \times 60.3 \mathrm{~cm}$, attaches directly onto the back of the da Vinci Surgical-System surgeon's console, so that the console can be used for VR training without the need for the patient's-side cart or instruments.

The exercises in the Mimic Technologies software are organized into systems-training and skills-training modules: EndoWrist manipulation, camera and clutching, system settings and console overview, needle control and driving, and energy and dissection. For the present study, seven exercises were selected for the training period: camera targeting level 2, pick and place, ring walk level 2, matchboard level 1 , needle targeting, suture sponge level 1 , dots and needles level 2. These seven exercises represent the diverse set of basic skills required for the performance of robotic surgery.

\section{Procedures}

The study design is represented in Figure 2. Participants initially conducted step-by-step exercises on the roboticdot platform and then the robotic skin-suturing pod using the da Vinci robot. Each exercise was performed twice to ensure the participants became familiar with it, but only the second performance was analysed. The performances
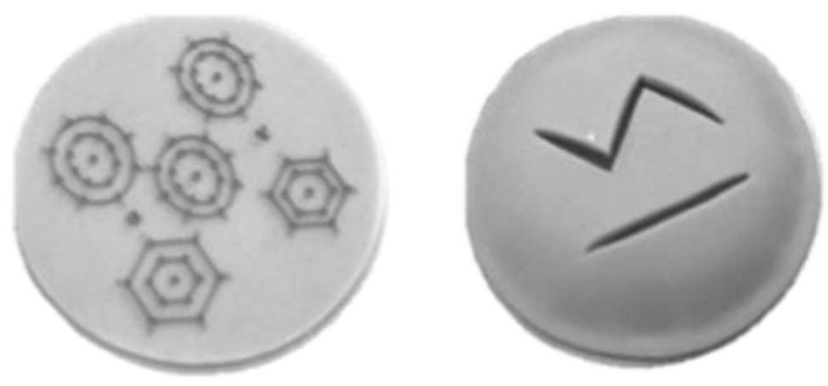

Figure 1. Robotic-dot and suturing-skin pod platforms 


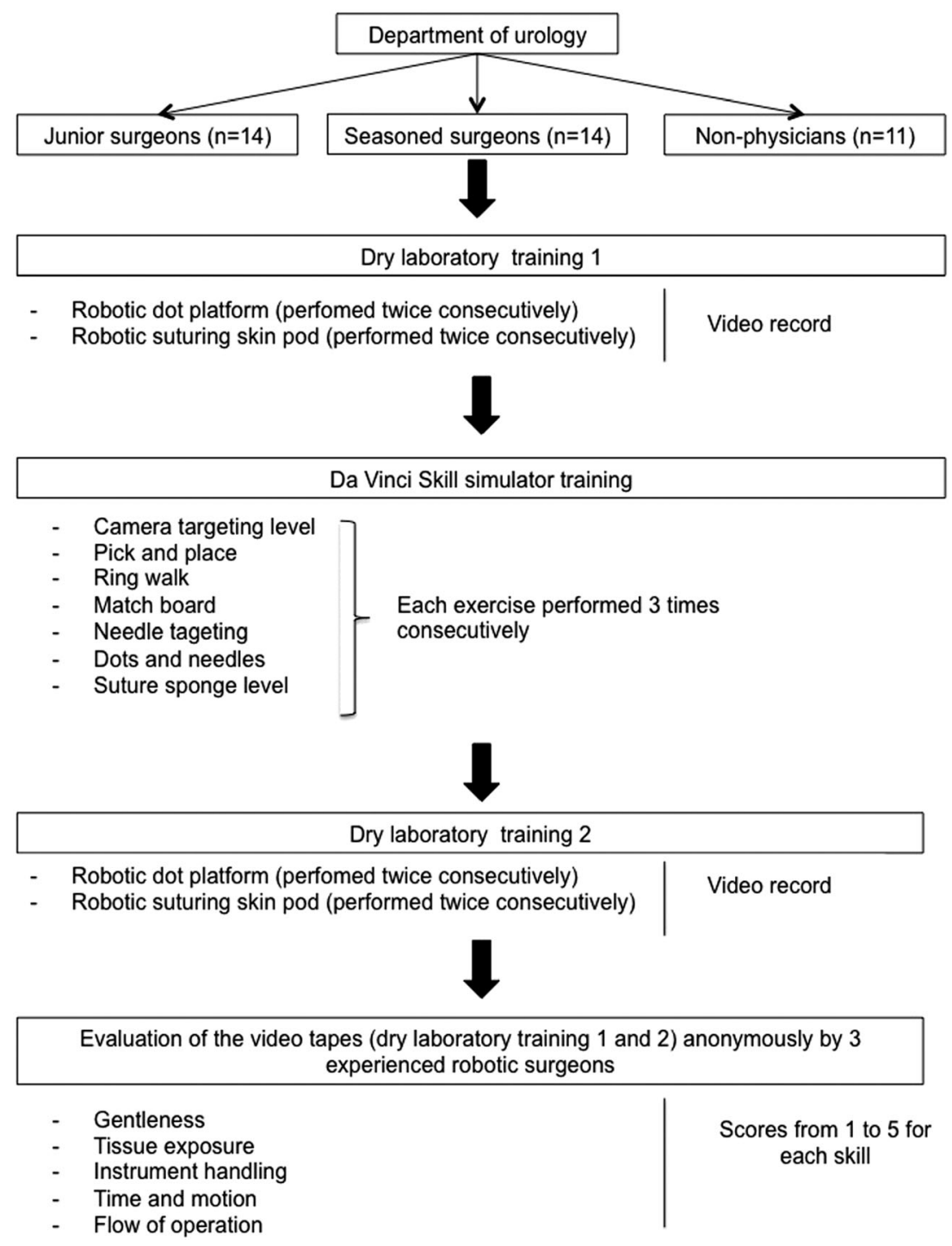

Figure 2. Study design

were recorded on a camera connected to the optical trocar of the da Vinci robot. Secondly, participants trained with the dVSS and performed the seven chosen exercises three consecutive times. Consecutively to the VR training, the participants returned to the robotic-dot and skin-suturing platforms and repeated the two exercises again. These performances were also recorded.

\section{Evaluation}

Three seasoned robotic surgeons (MR, JP, CV) evaluated, anonymously and independently, each videotape of the exercises on robotic-dot and suturing-skin pod platforms conducted before and after simulation training. They used a slightly modified tool from the Objective Structured Assessment of Technical Skills (14), which is commonly used to assess the skill of surgical trainees (2). Surgeonevaluators assessed each video according to five domains of technical skill: gentleness, tissue exposure, instrument handling, time and motion, and flow of operation. Each domain of surgical skill was rated on a scale from 1 to 5 .

\section{Outcome measures}

The primary outcome was improvement in surgical skill after the dVSS programme.

\section{Statistical analyses}

Scores are described with their means and standard deviations (SD). Student's t-test was used to compare performance metrics before and after the VR training-simulator 
programme. The Kruskal-Wallis test was used to compare performance metrics between the junior surgeons, the seasoned surgeons, and the non-physicians. When there was a significant difference, a Dunn's post-hoc test was conducted. A $P$-value of $<0.05$ was considered statistically significant for all analyses. Statistical analyses were performed using GraphPad Prism Software, version 6.

\section{Results}

Overall, 39 participants were enrolled in the study, with a mean age of 32.1 years (range: 22-62). In the juniorsurgeon group, 14 participants ( 5 women and 9 men) were included, mean age 27.5 years $( \pm 3.6)$. In the seasonedsurgeon group, 14 participants ( 2 women, 12 men) were included, mean age 42.4 years $( \pm 11.1)$. In the non-physician group, 11 participants (1 woman, 10 men) were included, mean age 25.5 years $( \pm 4.9)$. All participants completed the training programme in dry-laboratory platforms using the VR robotic simulator described previously.

The mean global scores for each exercise at the dVSS were significantly improved for each participant within each group between the first and the third trials. After VR robotic simulation training, the results from the robotic-dot platform were improved only for the nonphysicians and for the following items: respect for tissue, time and motion, instrument handling, tissue exposure and flow of operation (Table 1). The results before and after robotic-simulation training did not statistically differ for the junior or seasoned surgeons (Table 1).

Similarly, after VR robotic-simulation training, the results for the suturing-skin pod were improved only for non-physicians and for all the skill domains (Table 1). As above, the results from junior and seasoned surgeons did not change over time (Table 1 ).

Before VR robotic simulation training, the initial performances on the robotic-dot platform and the robotic suturing-skin pod were significantly lower in the group of non-physicians for each domain of technical skill and in comparison with the two other groups of participants. Similar results were reported after VR robotic simulation training.

\section{Discussion}

VR simulation is an emerging method used to train for robotic surgery, but appears to be still limited to basic-skills training.

In the present study, we report that a VR simulatortraining programme, using seven exercises of the dVSS, did not improve basic surgical skills when used on a drylaboratory platform in junior and seasoned surgeons.

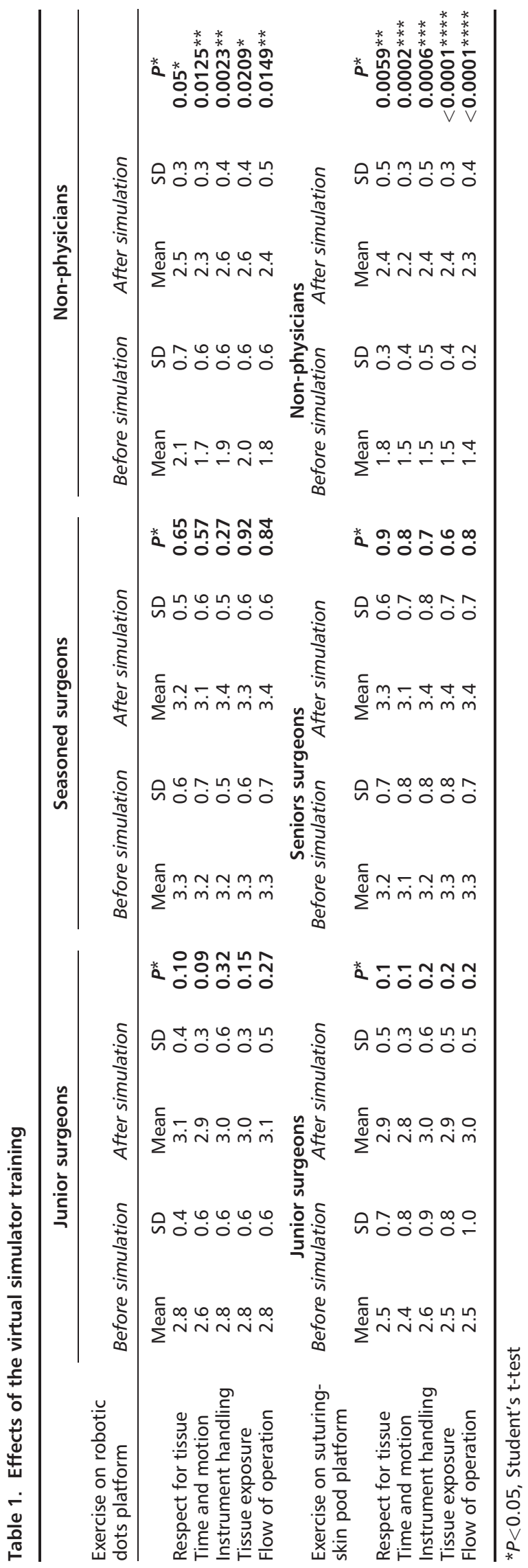


Surprisingly, the performances in the dry-laboratory platforms did not differ between junior and seasoned surgeons.

VR robotic simulation could be useful as a bridge for learners at the beginning of their learning curves. Those who have already acquired surgical experience may not benefit from this type of training (4). The validity of the dVSS has been evaluated by several reports $(4,12)$ and has been reported to be close to reality and useful (15). Nevertheless, its utility in improving skills during 'live' surgery has not been evaluated.

In the present study, we have demonstrated that skills were not increased after VR robotic simulation, especially for medical students and residents in training. From this perspective, our results conflict with those reported by another study in which the authors observed a significant improvement in performance and a reduction in errors among surgeons of varying experience after a VR warmup in basic robotic-surgical tasks (11). In addition, the VR warm-up reduced errors on a more complex task (robotic suturing), suggesting the generalizability of the warm-up. However, in that study, the authors used a different dry-laboratory platform from us. Moreover, to evaluate the objective performance metrics, they used a system consisting of recording by video and recording surgical-tool motion. In our study, we asked surgeons experienced in robotic surgery to anonymously evaluate videotapes using a tool that had been slightly modified from the Objective Structured Assessment of Technical Skills (14), which is commonly used to assess trainees' skills. We reported that the performances for the dot-platform/ suturing skin were statistically improved only for nonphysicians (who had no experience in robotic surgery), contrary to junior surgeons (who also had no experience in robotic surgery). These outcomes may be due to the fact that even though being junior, the junior-surgeons have been exposed to open surgery and know how to handle a needle or to perform a continuous straight-line suture and make knots. By contrast, non-physicians were completely naïve to any surgery.

We did not find any difference between junior and senior surgeons' skills in robotic surgery. However, this does not necessarily mean that young trainees who have mastered the dry-laboratory platform could translate their skills to real 'live' surgery. Unfortunately, most of the exercises currently available on VR simulators are generic tasks that test hand-eye co-ordination, tissue manipulation, suturing and knot tying. There is no evidence to assess which exercises improve real performances. Consequently, we can hypothesize that training on the dVSS and the dry-laboratory platforms does not reflect real surgical skills according to actual experience or 'live' surgery. Thus, the VR robotic simulator does not seem to prepare trainees to carry out robotic surgery unless they also have no prior knowledge about the surgery as reflected by our group of non-physicians. Ability to perform a robotic procedure depends on three elements: ability to safely and efficiently manipulate the robot, familiarity with the execution of surgical tasks for the procedure, and dissection skills. Training with a simulator can only ever equip a neophyte surgeon with the first element: how to manipulate a robot safely and efficiently. With increasing technological advancement in VR surgery, it would be possible in the future to equip novice surgeons with all elements required to perform a robotic operation. Until then, simulation will only have an initial benefit for novice surgeons.

It has been shown that simulation models are valid and reliable for the initial phase of training and evaluation of urological procedures; however, this is not the case for advanced and specialist-level skills (16). Our medical students and residents were already trained to carry out surgery, especially open surgery.

A further problem is the cost-effectiveness of a VR simulation programme. The cost of a robotic system alone is several million dollars, and the dVSS costs 89 000 US dollars (4). Therefore, the availability of such expensive equipment for training is usually low.

The main limitation of our study was its applicability to patients in 'live' surgery. Our findings were unambiguous in a dry-laboratory setting, but the true test is within the operating theatre. In contrast, animal and cadaver simulation models can simulate human anatomy, and these models are used in procedural training (2). Animal and cadaver models have been considered one of the most important components in robotic training programmes (1720 ) and are now incorporated into several courses. We acknowledge another limitation in our study in the fact that the participants performed seven exercises on the dVSS only for three consecutive times which did not allow us to assess if they achieved a plateau in their performances on the third repetition of the exercises.

In this study, the VR robotic-training skills were conducted on a dry-laboratory platform using the da Vinci Robot so that trainees were in a safe environment without the stress of live surgery. In addition, increased robotic experience does decrease complication rates in robotic prostatectomy (8). A junior robotic surgeon who is trained with an experienced surgeon and has experience with a real patient will learn more quickly.

During robotic surgery, surgeons perform procedures without tactile feedback and are physically distant from the operative field; this makes it challenging to learn each step of the procedure from a senior surgeon, compared with open or laparoscopic approaches.

The development of the dual-console da Vinci Si Surgical System $\AA$ has enabled modification to training practices. However, this teaching modality requires extra manpower: another assistant surgeon is needed at the patient's table to 
deal with the instruments via the assistant ports, and to exchange robotic instruments, etc. (21). With the dual-console setup, the proctoring surgeon and the proctored surgeon sit at the master and secondary consoles, respectively.

The use of a dual-console has been reported to create an integrated teaching and supervisory environment without compromising operative times or patient outcomes during gynaecologic surgery (22). Similarly, during radical prostatectomy, the dual-console system might improve intraoperative and perioperative outcomes (23). Indeed, compared with a single-console system, a significant decrease in mean operative time has been reported with the use of a dualconsole system as well as the incidence of intra-operative and post-operative complications. Outcomes on continence, erectile function, and the rate of biochemical recurrence did not differ between the use of a single and dual console. Thus, the use of a dual-console enables integrated teaching and surgical cooperation with proctoring. Evaluating a certified training programme for robotic surgery is currently needed. An international multidisciplinary group of experts proposed a multi-step curriculum for robotic training but further studies are required to validate the effectiveness of the curriculum and to evaluate transfer of skills to the operating room (24).

\section{Conclusion}

With the increasing popularity of robotic surgery there is a growing need for training programmes to overcome the learning curves inherent in this new surgical approach. From this perspective, the use of surgical simulation in residency programmes has expanded. Despite the initial interest, however, the VR robotic-simulator does not clearly improve surgeons' skills in robotic surgery in our initial and early experience with the simulator.

Alternative methods need to be considered to overcome the learning curves involved in robotic surgery, such as the use of a double-deck Da Vinci console during live surgery.

\section{Conflict of interest}

CV is a proctor surgeon for Intuitive surgical VP, SC, JP, MOB, VA, MR: No competing financial interests exist.

\section{References}

1. Buffi N, Van Der Poel H, Guazzoni G, Mottrie A. Junior European Association of Urology (EAU) Robotic Urology Section with the collaboration of the EAU Young Academic Urologists Robotic Section. Methods and priorities of robotic surgery training program. Eur Urol 2014; 65: 1-2.
2. Reznick RK, MacRae H. Teaching surgical skills - changes in the wind. N Eng J Med 2006; 355: 2664-2669.

3. Khan R, Aydin A, Khan MS, et al. Simulation-based training for prostate surgery. BJU Int 2014; 116: 665-674.

4. Abboudi $\mathrm{H}$, Khan MS, Aboumarzouk O, et al. Current status of validation for robotic surgery simulators - a systematic review. BJU Int 2013; 111: 194-205.

5. Seymour NE, Gallagher AG, Roman SA, et al. Virtual reality training improves operating room performance. Ann Surg 2002; 236: 458-464.

6. da Cruz JAS, Sandy NS, Passerotti CC, et al. Does training laparoscopic skills in a virtual reality simulator improve surgical performance? J Endourol Endourol Soc 2010; 24: 1845-1849.

7. Crochet P, Aggarwal R, Dubb SS, et al. Deliberate practice on a virtual reality laparoscopic simulator enhances the quality of surgical technical skills. Ann Surg 2011; 253: 1216-1222.

8. Lebeau T, Rouprêt M, Ferhi K, et al. The role of a well-trained team on the early learning curve of robot-assisted laparoscopic procedures: the example of radical prostatectomy. Int J Med Robot Comput Assist Surg 2011.

9. Thiel DD, Patel VR, Larson T, et al. Assessment of robotic simulation by trainees in residency programs of the southeastern section of the american urologic association. J Surg Educ 2013; 70: 571-577.

10. Schreuder H, Wolswijk R, Zweemer R, et al. Training and learning robotic surgery, time for a more structured approach: a systematic review. Int J Obstet Gynaecol 2012; 119: 137-149.

11. Lendvay TS, Brand TC, White L, et al. Virtual reality robotic surgery warm-up improves task performance in a dry laboratory environment: a prospective randomized controlled study. J Am Coll Surg 2013; 216: 1181-1192.

12. Hung AJ, Patil MB, Zehnder P, et al. Concurrent and predictive validation of a novel robotic surgery simulator: a prospective, randomized study. $J$ Urol 2012; 187: 630-637.

13. Moglia A, Ferrari V, Morelli L, et al. Distribution of innate ability for surgery amongst medical students assessed by an advanced virtual reality surgical simulator. Surg Endosc 2014; 28: 1830-1837.

14. Martin JA, Regehr G, Reznick R, et al. Objective structured assessment of technical skill (OSATS) for surgical residents. $\mathrm{Br} J$ Surg 1997; 84: 273-278.

15. Hung AJ, Zehnder P, Patil MB, et al. Face, content and construct validity of a novel robotic surgery simulator. $J$ Urol 2011; 186: 1019-1024.

16. Ahmed K, Jawad M, Abboudi M, et al. Effectiveness of procedural simulation in urology: a systematic review. J Urol 2011; 186: 26-34.

17. McDougall EM, Corica FA, Chou DS, et al. Short-term impact of a robot-assisted laparoscopic prostatectomy 'mini-residency' experience on postgraduate urologists' practice patterns. Int $J$ Med Robot Comput Assist Surg 2006; 2: 70-74.

18. Mehrabi A, Yetimoglu CL, Nickkholgh A, et al. Development and evaluation of a training module for the clinical introduction of the da Vinci robotic system in visceral and vascular surgery. Surg Endosc 2006; 20: 1376-1382.

19. Hanly EJ, Marohn MR, Bachman SL, et al. Multiservice laparoscopic surgical training using the daVinci surgical system. Am J Surg 2004; 187: 309-315.

20. Vlaovic PD, Sargent ER, Boker JR, et al. Immediate impact of an intensive one-week laparoscopy training program on laparoscopic skills among postgraduate urologists. J Soc Laparoendosc Surg 2008; 12: 1-8.

21. Fernandes E, Elli E, Giulianotti P. The role of the dual console in robotic surgical training. Surgery 2014; 155: 1-4.

22. Smith AL, Scott EM, Krivak TC, et al. Dual-console robotic surgery: a new teaching paradigm. $J$ Robot Surg 2013; 7: 113-118.

23. Morgan MSC, Shakir NA, Garcia-Gil M, et al. Single versus dualconsole robot-assisted radical prostatectomy: impact on intraoperative and postoperative outcomes in a teaching institution. World J Urol 2014.

24. Ahmed K, Khan R, Mottrie A, et al. Development of a standardised training curriculum for robotic surgery: a consensus statement from an international multidisciplinary group of experts. BJU Int 2014. 